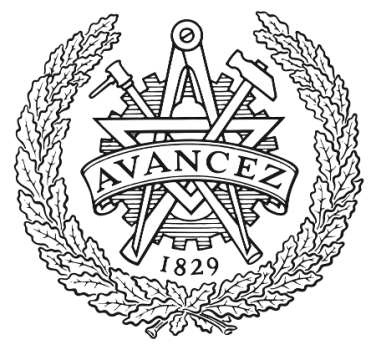

CHALMERS

UNIVERSITY OF TECHNOLOGY

\title{
Co-Design and Validation Approach for Beam-Steerable Phased Arrays of Active Antenna Elements with Integrated Power Amplifiers
}

Downloaded from: https://research.chalmers.se, 2023-04-26 14:48 UTC

Citation for the original published paper (version of record):

Vilenskiy, A., Liao, W., Maaskant, R. et al (2021). Co-Design and Validation Approach for Beam-Steerable Phased Arrays of Active Antenna Elements with Integrated Power Amplifiers. IEEE Transactions on Antennas and Propagation, 69(11): 7497-7507. http://dx.doi.org/10.1109/TAP.2021.3076255

N.B. When citing this work, cite the original published paper.

(C2021 IEEE. Personal use of this material is permitted.

However, permission to reprint/republish this material for advertising or promotional purposes 


\title{
Co-Design and Validation Approach for Beam-Steerable Phased Arrays of Active Antenna Elements with Integrated Power Amplifiers
}

\author{
Artem. R. Vilenskiy, Member, IEEE, Wan-Chun Liao, Student Member, IEEE, Rob Maaskant, Senior \\ Member, IEEE, Vessen Vassilev, Member, IEEE, Oleg A. Iupikov, Member, IEEE, Thomas \\ Emanuelsson, Member, IEEE, Marianna V. Ivashina, Senior Member, IEEE
}

\begin{abstract}
An approach for designing a beam-steering active phased array of antenna elements with integrated power amplifiers (PAs) is presented. It is based on an amplifying active integrated unit cell (AiUC) concept, where the AiUC comprises a radiating slot element, a GaN high electron mobility transistor (HEMT), its input matching and DC biasing/feeding circuitry. The HEMT is embedded in the antenna element, being directly impedance-matched to HEMT's drain output, i.e. without using any intermediate and potentially lossy impedance matching network. The proposed co-design approach involves a full-wave analysis of the AiUC passive part (naturally including elements mutual coupling effects) along with the subsequent fullsystem harmonic balance simulations. Furthermore, we extend the standard definition of the scan element pattern (SEP) to the active scan element pattern (ASEP) that accounts for nonlinear effects of PAs on AiUC performance. We show that the ASEP is, in general, power-dependent and has a different shape as compared to the SEP. The proposed approach has been demonstrated for a K-band AiUC design example. It was verified through an active waveguide simulator, which is equivalent to the 23.7 $\mathrm{H}$-plane beam-steering case. Measurements are in good agreement with simulations, revealing AiUC $47 \%$ peak drain efficiency and $33 \mathrm{dBm}$ maximum radiated power. The predicted scan range is $\pm 60^{\circ}$ and $\pm 37^{\circ}$ in the $\mathrm{E}$ - and $\mathrm{H}$-plane, respectively.
\end{abstract}

Index Terms-active antenna, array antenna, beam steering

\section{INTRODUCTION}

Amplifying active integrated antenna (AiA) designs have been widely studied [1], [2], where the antenna typically represents an integrated combination of a radiating element and an active device (power amplifier, PA). These designs can provide high radiated power, high power efficiency, and compact size, as compared to conventional active antenna implementations. The benefits of AiAs become more noticeable and vital for millimeter-wave (mm-wave) frequency applications, where

Manuscript received $\mathrm{xx} \mathrm{xx}, 20 \mathrm{xx}$; revised $\mathrm{xx} \mathrm{xx}, 20 \mathrm{xx}$. This research has been carried out in ChaseOn Centre in Integrated Antenna Array project financed by Vinnova, Chalmers University of Technology, KTH, Ericsson, Saab, Ruag Space, Keysight, and Gapwaves. (Corresponding author: A. R. Vilenskiy)

A. R. Vilenskiy, W.-C. Liao, O. A. Iupikov, and M. V. Ivashina are with the Electrical Engineering Department, Chalmers University of Technology, 41296 Gothenburg, Sweden (e-mail: artem.vilenskiy@ chalmers.se)

R. Maaskant is with the Electrical Engineering Department, Chalmers University of Technology, and also with the Department of Electrical Engineering, Eindhoven University of Technology, 5612 AZ Eindhoven, The Netherlands.

V. Vassilev is with the Microwave Electronics Laboratory, Chalmers University of Technology, 41296 Gothenburg, Sweden.

T. Emanuelsson is with Ericsson AB, Lindholmspiren 11, 41756 Gothenburg. Sweden. dissipative losses of printed transmission lines and antennaPA interconnections can be severe. The state-of-the-art in mmwave AiA systems covers several design implementations. In [3]-[6], a PA is combined with an antenna element either on a single printed circuit board (PCB) or within a PCB assembly to maximize power efficiency and radiated power at S-/C-bands. This approach is, however, not well suited for higher frequencies, due to the increasing power losses of matching circuits and harmonic filters introduced between the PAs and antennas. To overcome this problem, direct impedance matching between the PA and radiating element can be used, as was demonstrated in [7], [8] for PCB-based designs and [9] for the antenna-in-package architecture. Monolithic microwave integrated circuits (MMICs) is a popular alternative, offering compact design and high repeatability (see [10], [11]). A common drawback is low radiation efficiency of usually narrowband on-chip antennas, especially at mm-wave frequencies.

The above-described enhancements have been demonstrated using rather conventional antenna integration approaches, where the radiator has a well-defined excitation port that is interconnected with an active device through intermediate circuitry. Further increasing the integration density is possible [12]-[14], e.g., by embedding active components inside the radiator and impedance matching it directly to the optimum PA load impedance. The optimum PA load impedance is commonly defined for the maximum power efficiency or maximum gain and is generally complex-valued, hence rather different from the standard $50 \Omega$. Recently, this concept of direct integration and impedance matching was reported in [15] for a K-band GaN high electron mobility transistor (HEMT)-integrated cavity-backed slot antenna. This antenna, as well as all the above-mentioned AiAs, has been designed as an isolated element, hence without considering the effects of antenna mutual coupling and nonlinearity of PAs in arrays of such AiA elements. Nevertheless, the considered direct integration approach can be further extended to the development of active integrated array antennas (AiAAs). Such AiAAs are potentially capable of providing a wide-angle 2D (full-space) beam-steering range with high effective isotropic radiated power. The design of an active integrated element for array antenna applications is a significant challenge. In this work, we will refer this element to as an active integrated unit cell (AiUC). It should be noted that different designs of amplifying AiAAs have been previously considered [3], [16]-[20]. In [16] 


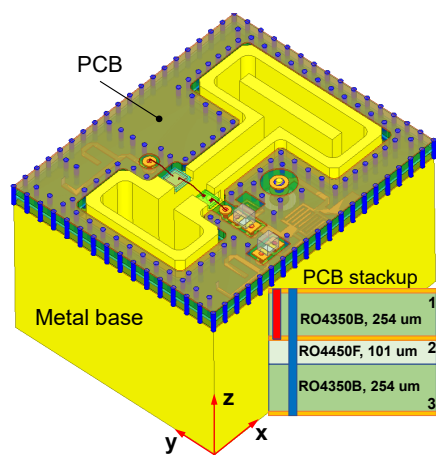

(a)

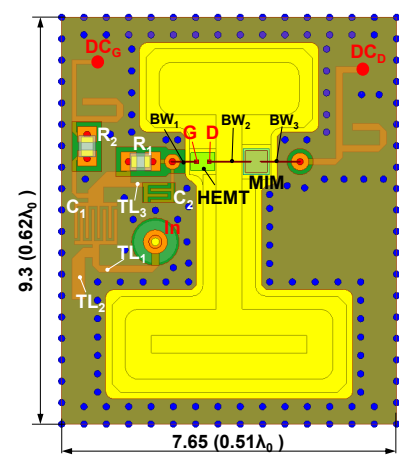

(b)
Fig. 1. The AiUC configuration: (a) perspective view (the PCB stackup is given in the inset), (b) top view (all dimensions are in $\mathrm{mm}$ ).

and [18], an integrated $2 \times 2$ MMIC sub-array at Q-band and a fixed-beam C-band AiAA were reported, respectively, where the radiator and the PA are physically separated with the 50$\Omega$ interstage impedance matching networks. Another concept was presented in [20], where a unified deep-integrated AiA was used in a 1D amplifier-circuit-antenna array. This concept fully obviates the need in any input/output matching circuitry. Unfortunately, it is currently applicable only to the series-fed 1D AiAs with minor beam steering.

This paper presents a further development of the work in [15] towards wide-angle 2D beam-steerable AiAAs of AiUCs. We focus on the AiUC in the infinite array environment that leads to the antenna element design for large-scale AiAAs. The key novel contributions are three-fold: (i) an AiUC co-design methodology based on the infinite array electromagnetic (EM)circuit co-simulations and a new AiUC beam-steering performance metric, referred to as an active scan element pattern (ASEP), which jointly account for the antenna mutual coupling and nonlinear effects of PAs in the array design process; (ii) an AiUC design example with an embedded GaN HEMT at Kband, optimized through the proposed co-design methodology; (iii) an experimental validation setup for the AiUC that is based on a two-element active waveguide simulator concept. Its performance is equivalent to the $23.7^{\circ} \mathrm{H}$-plane beamsteering infinite array case and is used to extract the ASEP.

The paper is organized as follows. Section II presents the proposed co-design approach through an example AiUC. The ASEP is defined in Section III. Section IV addresses the design of the two-element active waveguide simulator. Section V provides results discussion and comparison with prior-art. Finally, the conclusions are formulated in Section VI.

\section{Co-Design Approach FOR An AiUC}

\section{A. AiUC Description}

The AiUC was developed for a planar uniform array with a rectangular lattice at the central frequency of $f_{0}=20 \mathrm{GHz}$. Its $3 \mathrm{D}$ view is given in Fig. 1. This AiUC takes the element type in [15] as the starting point, where we have introduced several significant modifications to make the design suitable for beamsteering array applications, i.e. to optimally perform in the presence of antenna elements mutual coupling and nonlinear

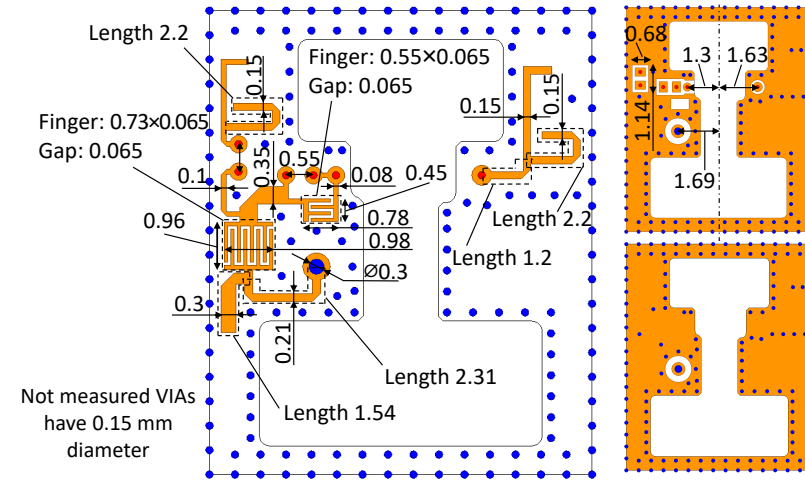

Fig. 2. PCB layers topologies with main design dimensions (in $\mathrm{mm}$ ): left - enlarged layer 2 (inner), top right - layer 1 (top), bottom right - layer 3 (bottom). Layer numbers are given in Fig. 1(a). Through VIAs (blue) connect all PCB layers; blind VIAs (red) connect layer 1 and layer 2.

effects of PAs. Another important aspect is to make it suitable for the verification through the active waveguide simulator approach as detailed in Section IV.

The AiUC comprises the brass base which supports the 3-layer PCB. The asymmetrical H-shaped cavity-backed slot radiator is milled in the base. The lower $y$-oriented slot segment [Fig. 1(b)] incorporates the ridge element to increase the equivalent slot electrical length while preserving its overall dimensions. The straight $x$-oriented slot segment gives the major contribution to the radiated field defining the radiation resistance, whereas the $y$-oriented parts contribute to the antenna reactance. The slot element has $x z$-plane symmetry, which provides a well-defined polarization plane ( $y z$-plane). A HEMT is mounted directly at the slot edge [Fig. 1(a)]. Its gate port is connected via the bondwire $B W_{1}$ to the PCB. The bondwire $B W_{2}$ transversely crosses the slot and connects the HEMT's drain to the shunt 2.2-pF MIM capacitor (Tecdia BMS2R2J1K), thus directly exciting the radiating element. The drain DC feed is applied via the bondwire $B W_{3}$. In this design, as well as in [15], we employed the GaN HEMT TGF2942 from Qorvo. Moving the HEMT along the $x$-axis we can effectively tune the antenna impedance visible from the drain side. The PCB stackup is given in the inset of Fig. 1(a). The PCB is formed by two 254- $\mu$ thick Rogers RO4350B cores bounded by $101-\mu \mathrm{m}$ thick Rogers RO4450F prepreg. The topologies of the three conducting layers are detailed in Fig. 2. The AiUC is excited through the 50- $\Omega$ orthogonal coaxial assembly (2.92 mm connector 1014-21SF with 1090-11G launching pin from Southwest). Two stripline segments $T L_{1}$ and $T L_{2}$ [Fig. 1(b)] form the input matching circuit connected to the eight-fingers interdigital DC blocking capacitor $C_{1}$. The gate stabilization resistor $R_{1}=50 \Omega$ is connected in parallel with the combination of $T L_{3}$ cascaded with the four-finger interdigital capacitor $C_{2}$. This circuit is tuned to have the series resonance at $f_{0}$. The equivalent AiUC input circuit representation is, in general, similar to the one given in [15]. An additional high-frequency resistor $R_{2}=25 \Omega$ was introduced inside the gate biasing line to improve the low-frequency AiUC stability. Apart from this, the important distinction with the design from [15] is the input circuitry shielding realized by the metallization of the top PCB layer. 


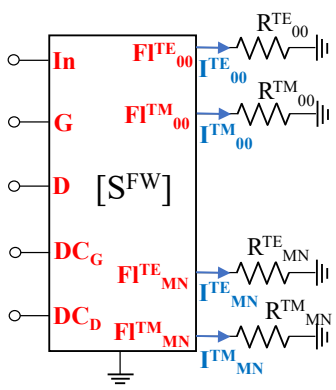

(a)

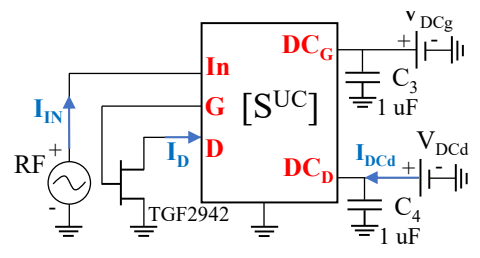

(b)
Fig. 3. (a) The 5-port $S$-matrix $S^{U C}$ derived from $S^{F W}$ by the Floquet modal ports termination. $S^{F W}$ is computed using the $3 \mathrm{D}$ full-wave model (Fig. 1). (b) Circuit diagram of the harmonic balance co-simulation setup.

The AiUC transverse dimensions are $d_{x}=0.62 \lambda_{0}, d_{y}=$ $0.51 \lambda_{0}$, where $\lambda_{0}$ is the free-space wavelength corresponding to $f_{0}$. This allows having the grating lobes-free beam-steering regime up to $74^{\circ}$ in the E-plane (yz-plane) and up to $38^{\circ}$ in the H-plane ( $x z$-plane).

\section{B. Co-Design Methodology}

The proposed AiUC comprises both linear (passive) and nonlinear (active) parts. The AiUC nonlinear effects become significant when the HEMT is driven with a large input signal and operates in class-AB [21], which is the case when both high efficiency and linearity are required. A combined circuitEM co-simulation approach was developed to analyze the AiUC. The linear passive AiUC part was simulated in Ansys HFSS. The five AiUC ports are depicted in Fig. 1(b): In (coaxial input), $G$ (HEMT gate), $D$ (HEMT drain), $D C_{G}$ (gate DC biasing), $D C_{D}$ (drain DC feeding). Since the HEMT is electrically small, we assume that its interaction with the PCB circuitry and radiating structure can be correctly described through two lumped ports $G$ and $D$. The model sidewalls boundary conditions were set to periodic, and the Floquet port was introduced at $30 \mathrm{~mm}$ above the AiUC. Thus, all array elements mutual coupling effects are naturally included through the AiUC boundary conditions. The Floquet port contains $N_{F}$ modes, which represent Floquet $T E_{m n}$ and $T M_{m n}$ modes, where $m, n$ are modal indices [22]. This EM model provides the $S$-matrix $S^{F W}$ that fully describes all interactions inside the linear AiUC part. Next, $S^{F W}$ is transferred to Keysight ADS environment, where it is reduced to the 5-port $S$-matrix $S^{U C}$ by terminating the Floquet modal ports with matched loads $R_{m n}^{T E / T M}$ [Fig. 3(a)].

Next, the 5-port $S^{U C}$ was used in the ADS harmonic balance (HB) simulator according to the setup in Fig. 3(b). The applied DC conditions are: $V_{D C g}=-2.4 \mathrm{~V}, V_{D C d}=28 \mathrm{~V}$, $I_{D C d}=35 \mathrm{~mA}$. The HB convergence has been reached with the first three harmonics, hence the full-wave analysis of the AiUC was performed up to $3 f_{0}$. For accurate analysis, the Floquet port includes all modes that can propagate for the full hemispherical beam-steering sector at $3 f_{0}$. This results in $N_{F}=30$, and $S^{F W}$ represents the 35-port $S$-matrix.

During beam steering, $S^{F W}$ changes for each beam direction $\left(\theta_{s}, \varphi_{s}\right)$, where $\theta_{s}, \varphi_{s}$ are elevation and azimuth beamsteering angles, respectively. To speed up the simulations, a unified control environment was developed in MATLAB using MATLAB-HFSS and MATLAB-ADS interfaces. . The following AiUC characteristics are obtained from the cosimulation process: active drain impedance and reflection coefficient $Z_{D}, \Gamma_{D}$; active input impedance and reflection coefficient $Z_{i n}, \Gamma_{i n}$; transducer and available co- and crosspolarized power gains $G_{T}^{c / x}, G_{P}^{c / x}$; drain power efficiency $\eta_{D}$. It is worth noting that power gains are computed using power of the dominant Floquet modes $\left(P_{00}^{c / x}\right)$ related to co- and crosspolarized components:

$$
G_{T}^{c / x}=P_{00}^{c / x} / P_{a v s}, \quad G_{P}^{c / x}=P_{00}^{c / x} / P_{i n},
$$

where $P_{a v s}$ and $P_{i n}=P_{a v s}\left(1-\left|\Gamma_{i n}\right|^{2}\right)$ are the available from the source and input powers, respectively [21]. For each AiUC port, a reference impedance $Z_{0}$ can be, in general, complex [23]. The designations of all branch currents and nodal voltages can be found in Fig. 3(a) and Fig. 3(b).

The AiUC co-design aims at the maximum power efficiency by optimizing the HEMT loading conditions. The optimum HEMT drain impedance $Z_{\text {Dopt }}=17+j 46 \Omega$ was reported in [15] based on the load-pull analysis. Note that this value is not equal to the impedance $Z_{D c o n j}=11+j 44 \Omega$ providing the drain conjugate match. At the same time, the AiUC input $50-\Omega$ port should be matched with the HEMT input impedance from the gate side $Z_{T g}=3-j 13.2 \Omega$. Owing to the shielding of the input circuitry, at the first design stage the input matching circuit and the radiating slot structure can be tuned independently to provide $S_{D, D}^{F W}=\Gamma_{\text {Dopt }}$ and $S_{G, G}^{F W}=\Gamma_{G o p t}$, where $\Gamma_{\text {Dopt }}$ and $\Gamma_{\text {Gopt }}$ correspond to $Z_{\text {Dopt }}$ and $Z_{T g}^{*}$. Next, the obtained $S^{F W}$ is used in the HB simulator. The final AiUC structure optimization is performed iteratively.

The AiUC was optimized according with the proposed codesign methodology for the broadside beam. The main final AiUC structure parameters are: $x$-oriented slot segment $0.3 \times 0.04 \times 0.34 \lambda_{0}^{3}$; upper $y$-oriented slot segment $-0.08 \times$ $0.21 \times 0.34 \lambda_{0}^{3}$, lower segment $-0.11 \times 0.32 \times 0.34 \lambda_{0}^{3}$; ridge $-0.027 \times 0.24 \times 0.34 \lambda_{0}^{3}$; HEMT shift relative to the AiUC center $-0.09 \lambda_{0}$. The slot is allocated inside the AiUC in such a way that its edges are equally spaced from the AiUC $x$ boundaries. The main PCB dimensions are presented in Fig. 2.

\section{Broadside Performance}

The broadside AiUC performance was studied first by assigning $\left(\theta_{s}, \varphi_{s}\right)=(0,0)$. Simulated AiUC small-signal characteristics are presented in Fig. 4(a). The peak $G_{T}^{c}=8.4 \mathrm{~dB}$, whereas the relative cross-polarization level is below $-30 \mathrm{~dB}$. The simple 2-element matching circuit provides a relatively narrowband matching with almost pure reactive HEMT input. As a result, the $3-\mathrm{dB} G_{T}^{c}$ bandwidth is $540 \mathrm{MHz}$. However, the $G_{P}^{c}$ curve indicates a wider available gain bandwidth of $820 \mathrm{MHz}$.

Next, we explored the large-signal performance within the $20 \pm 0.25 \mathrm{GHz}$ bandwidth by sweeping $P_{\text {avs }}$ in the $-10 \ldots 35 \mathrm{dBm}$ range. Fig. 4(b) demonstrates simulated $\Gamma_{D}$ at fundamental and harmonics frequencies. As expected, $\Gamma_{D}$ does not depend on $P_{a v s}$ due to the small coupling between the AiUC radiating parts and the input circuitry. The results 


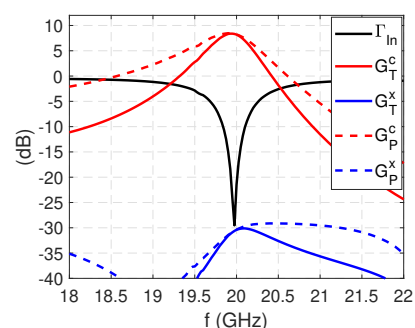

(a)

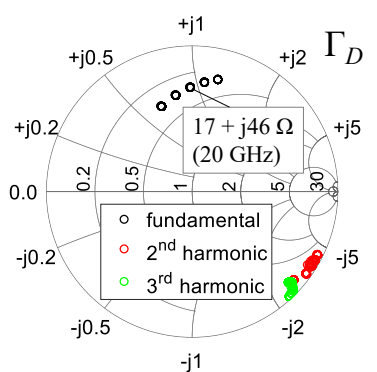

(b)
Fig. 4. (a) Simulated small-signal performance of the AiUC in the broadside regime: (b) Simulated active drain reflection coefficient $\Gamma_{D}$ at fundamental $\left(20 \pm 0.25 \mathrm{GHz}, 0.125 \mathrm{GHz}\right.$ step) and harmonic frequencies. $P_{a v s}=$ $-10 \ldots 35 \mathrm{dBm} . Z_{0}=50 \Omega$.

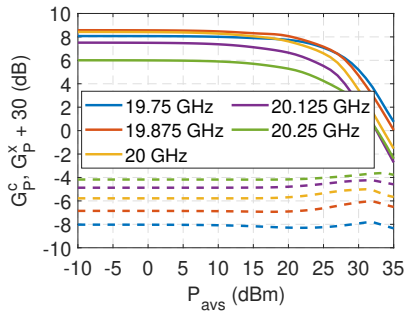

(a)

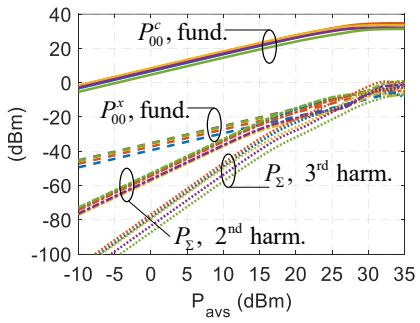

(b)

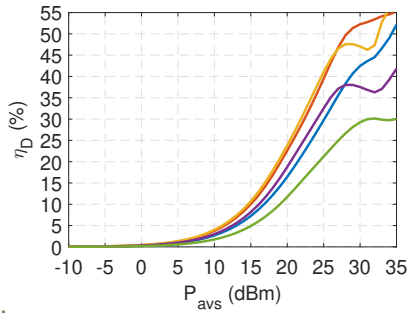

(c)

Fig. 5. Simulated large-signal broadside performance of the AiUC in the infinite array environment. (a) co- (solid lines) and cross-polarized (dashed lines) power gain of the AiUC; (b) radiated power at fundamental frequencies for co- and cross-polarized components separately, and full radiated power $\left(P_{\Sigma}\right)$ at the second and third harmonics; (c) drain efficiency.

evidence that the targeted $Z_{\text {Dopt }}$ was achieved at $f_{0}$, while $\Gamma_{D}$ amounts to $0.97 \exp \left(-j 32^{\circ}\right)$ and $0.97 \exp \left(-j 44^{\circ}\right)$ at $2 f_{0}$ and $3 f_{0}$, respectively. Maximum $\eta_{D}=59 \%$ can be theoretically realized with such harmonic terminations.

The gain performance is depicted in Fig. 5(a) for both coand cross-polarized components. The 1-dB compression point at $20 \mathrm{GHz}$ is $P_{1 d B}=21 \mathrm{dBm}$. Fig. 5(b) demonstrates the radiated powers at fundamental and harmonic frequencies. Each harmonic power $P_{\Sigma}$ was computed by summing the power of all propagating Floquet modes. The simulated $\eta_{D}$, presented in Fig. 5(c), predicts achievable $47 \%$ for $P_{a v s}=$ $27 \mathrm{dBm}$ at $20 \mathrm{GHz}$.

\section{BeAm-SteEring Performance of THE AiUC}

In the analysis of AiUCs, the radiating structure and the active component are closely integrated, with no well-defined "antenna port". This makes it difficult to characterize the radiating and active AiUC parts separately. Therefore, a new AiUC performance metric taking into account all the effects occurring in the integrated antenna-PA structure during beam steering is proposed.

\section{A. Active Scan Element Pattern of the AiUC}

The derivation of the classical scan element pattern (SEP) employs either the superposition [22], [24] or the reciprocity principle [25], but neither can be applied to the AiUC due to its nonlinear and nonreciprocal nature. The former property leads to a beam-steering performance dependence on the AiUC input power. Strictly speaking, for the AiUC we cannot introduce the standard SEP, which represents a far field of a single excited array element, while all other elements are terminated with matched loads. This is because in a nonlinear operation regime the full AiAA radiated field, in general, cannot be represented as a superposition of partial fields from separately excited AiUCs. Nevertheless, we still can characterize the radiation performance of the AiUC employing its equivalent powerdependent ASEP. To get the desired ASEP, let us consider the transverse electromagnetic field $(\mathbf{E}, \mathbf{H})$ at $f_{0}$ on the $x y$-plane at $z=z_{0}$ above the infinite AiAA aperture:

$$
\begin{aligned}
\mathbf{E} & =\sum_{(m, n)} A_{m n}^{T E} \mathbf{E}_{m n}^{T E}+\sum_{(m, n)} A_{m n}^{T M} \mathbf{E}_{m n}^{T M} . \\
\mathbf{H} & =\sum_{(m, n)} A_{m n}^{T E} \mathbf{H}_{m n}^{T E}+\sum_{(m, n)} A_{m n}^{T M} \mathbf{H}_{m n}^{T M},
\end{aligned}
$$

where $\mathbf{E}_{m n}^{T E / T M}, \mathbf{H}_{m n}^{T E / T M}$ are electric and magnetic field vectors of TE and TM Floquet modes [22]; $A_{m n}^{T E / T M}$ are constant modal amplitudes that depend only on $\left(\theta_{s}, \varphi_{s}\right)$ and $P_{\text {avs }}$. The detailed description of the modal fields is given in Appendix A. Note that for the chosen modal normalization (A.1), (A.2) $\left|A_{m n}^{T E / T M}\right|^{2} / 2=P_{m n}^{T E / T M}$.

The infinite array far field will still represent the superposition of the propagating Floquet modes (2), (3) with $A_{m n}^{T E / T M}$ multiplied by the corresponding phase factors. However, we will use another representation, introducing equivalent electric $\mathbf{J}_{m n}^{T E / T M}=\mathbf{z}^{0} \times \mathbf{H}_{m n}^{T E / T M}$ and magnetic $\mathbf{M}_{m n}^{T E / T M}=$ $-\mathbf{z}^{0} \times \mathbf{E}_{m n}^{T E / T M}$ surface currents, where $\mathbf{z}^{0}$ is the $z$-axis unit vector. Next, employing the far-field representation through the radiation integrals [26], we arrive at $\mathbf{E}_{F F m n}^{T E / T M}$ :

$$
\mathbf{E}_{F F m n}^{T E / T M}(\mathbf{r})=A_{m n}^{T E / T M} \mathbf{C}_{m n}^{T E / T M} \frac{\exp (-j k r)}{4 \pi r} I_{m n}(\theta, \varphi),
$$

where $\mathbf{r}$ is the radius vector of the field observation point with spherical coordinates $(r, \theta, \varphi)$. The derivation of (4) and definition of $\mathbf{C}_{m n}^{T E / T M}$ are detailed in Appendix A. The term $I_{m n}$ has the following form:

$$
\begin{aligned}
I_{m n}(\theta, \varphi) & =S \operatorname{sinc}\left(\left(k_{x}-k_{x m}\right) \frac{d_{x}}{2}\right) \operatorname{sinc}\left(\left(k_{y}-k_{y n}\right) \frac{d_{y}}{2}\right) \\
& \times \sum_{q=-\infty}^{\infty} \sum_{p=-\infty}^{\infty} \mathrm{e}^{j\left(k_{x}-k_{x 0}\right) p d_{x}+j\left(k_{y}-k_{y 0}\right) q d_{y}},
\end{aligned}
$$

where $k_{x}=k \sin (\theta) \cos (\varphi), \quad k_{y}=k \sin (\theta) \sin (\varphi)$, and $S=d_{x} d_{y}$ is the AiUC area. The double summation in (5) is the array factor (AF) with the beam-steering direction $\left(\theta_{s}, \varphi_{s}\right)$. It can be written as [22] 


$$
A F(\theta, \varphi)=\frac{4 \pi^{2}}{S} \sum_{q=-\infty}^{\infty} \sum_{p=-\infty}^{\infty} \delta\left(k_{x}-k_{x p}\right) \delta\left(k_{y}-k_{y q}\right) .
$$

In (6), the Dirac delta functions evidence that the AiAA radiates in the discrete number of angular directions, which was expected from (2), (3). During beam steering, the direction of interest is $\left(\theta_{s}, \varphi_{s}\right)$, i. e. $k_{x}=k_{x 0}, k_{y}=k_{y 0}$ (Appendix A). With this substitution into (4), we can notice that only $I_{00}$ is non-zero, and $C_{00 \theta}^{T E}=C_{00 \varphi}^{T M}=0$. Then, separating the $\mathrm{AF}$ in (4), we extract the contribution to the AiAA far field from a single AiUC ( $\left.\mathbf{E}_{a}\right)$, operating inside the fully excited (with a given $P_{\text {avs }}$ ) array environment:

$\mathbf{E}_{a}\left(r, \theta_{s}, \varphi_{s}\right)=j \frac{\sqrt{\eta S \cos \left(\theta_{s}\right)}}{\lambda}\left(A_{00}^{T M} \boldsymbol{\theta}^{0}-A_{00}^{T E} \boldsymbol{\varphi}^{0}\right) \frac{\mathrm{e}^{-j k r}}{r}$,

where $\eta=120 \pi \Omega$ is the free-space wave impedance; $\theta^{0}$ and $\varphi^{0}$ are the $\theta$ - and $\varphi$-axis unit vectors, respectively. The angular dependence of $\mathbf{E}_{a}$ gives us the desired vector field ASEP. We now define the vector gain ASEP of the AiUC $\left(\mathbf{G}_{a}\right)$ following the standard definition of realized gain. Recalling that $A_{00}^{T E / T M}$ depends on $P_{a v s}$ and changing $\left(\theta_{s}, \varphi_{s}\right)$ to $(\theta, \varphi)$, we can express the gain ASEP in the following form

$$
\begin{array}{r}
\mathbf{G}_{a}\left(\theta, \varphi, P_{\text {avs }}\right)=\frac{4 \pi S \cos (\theta)}{\lambda^{2}}\left(G_{T}^{\theta}\left(\theta, \varphi, P_{\text {avs }}\right) \theta^{0}+\right. \\
\left.G_{T}^{\varphi}\left(\theta, \varphi, P_{\text {avs }}\right) \boldsymbol{\varphi}^{0}\right) .
\end{array}
$$

We can point out that the introduced ASEP comprises the AiUC transducer gains $G_{T}^{\theta / \varphi}=P_{00}^{T M / T E} / P_{\text {avs }}$, which are related to the amplifying property of the integrated PA, and the remaining part, similar to the standard SEP of a passive unit cell (UC) [22], related to the radiating element itself. Thus, the ASEP naturally incorporates both traditional antenna and PA effects. The latter includes PA gain compression and sensitivity to the load variation. It is the ASEP that should be used when the active gain $\mathbf{G}_{A A}$ of a large (negligible edge effects) AiAA of $N$ AiUCs to be computed, i.e. $\mathbf{G}_{A A}=N \mathbf{G}_{a}$ [24].

\section{B. Simulation Results}

The AiUC performance at $f_{0}$ for beam steering in the Eand H-planes is presented first. In Fig. 6(a), the co-polarized ASEPs are demonstrated for different $P_{\text {avs }}:-10 \mathrm{dBm}$ (linear regime) and $27 \mathrm{dBm}$ (2.5-dB compression). The conventional SEP of the passive UC radiating part is given for reference and defined as in [22]:

$\mathbf{G}_{U C}(\theta, \varphi)=\frac{4 \pi S \cos (\theta)}{\lambda^{2}}\left(\left|S_{T M 00, D}^{F W^{\prime}}\right|^{2} \boldsymbol{\theta}^{0}+\left|S_{T E 00, D}^{F W^{\prime}}\right|^{2} \boldsymbol{\varphi}^{0}\right)$,

where we employed renormalized transmission coefficients from the drain port to $T E_{00}$ and $T M_{00}$ Floquet modes. The renormalized $S$-matrix $S^{F W^{\prime}}$ was computed from $S^{F W}$ [27] with the following port reference impedances replacement $\left\{D, G, D C_{D}, D C_{G}\right\}=\{17-j 46,3-j 13.2,0.5,0.5\} \Omega$. The PA gain compression can be seen in 6(a) as the variation of the ASEP peak level. In the E-plane, the scan blindness nearby the appearance of the array grating lobe at $\theta \approx 73^{\circ}$ is observed. Meanwhile, the H-plane beam steering does not lead to scan blindness. It is useful to examine the normalized $G_{T}^{c}$ and $S_{T E / T M 00, D}^{F W^{\prime}}$, to understand the AiUC behavior with no accounting for the cosine aperture projection effect and different gain peak levels. These results are presented in Fig. 6(b). Drain efficiency dependencies on $\theta_{s}$ in the E- and H-plane are given in Fig. 6(c). We can observe that, even in the linear regime, the angular dependency of the $G_{T}^{c}$ differs from $S_{T E / T M 00, D}^{F W^{\prime}}$. We could expect this, as the radiating element is not conjugately matched with the HEMT drain (Section II-B). As a result, e.g. during beam steering in the E-plane, $Z_{D}$ quickly recedes from $Z_{D c o n j}=11+j 44 \Omega$ [Fig. 6(c)], and $G_{T}^{c}$ drops faster with $\theta_{s}$ compared to $S_{T M 00, D}^{F W^{\prime}}$. At the same time, in the H-plane, the $\Gamma_{D}$ locus is closer to the conjugately matching conditions, and thus the slopes of $G_{T}^{c}$ and $S_{T E 00, D}^{F W^{\prime}}$ curves are very close. Another important observation is that the angular shape of the ASEP depends on the power level, as can be clearly seen in Fig. 6(b). This is the result of the nonlinear HEMT behavior leading to the HEMT drain input impedance variation with $P_{\text {avs }}$. The two aforementioned phenomena, namely the discrepancy between SEP and ASEP angular shapes and the ASEP power-dependent nature, are the main distinctive features of the AiUC beamsteering performance as compared to the passive UC.

It is worth mentioning that $\Gamma_{i n}$ remains almost constant during beam steering owing to the decoupling of the input circuitry and the AiUC radiating elements. At the same time, the relative cross-polarization level is lower than $-27 \mathrm{~dB}$ and $-30 \mathrm{~dB}$ for the $\pm 60^{\circ}$ and $\pm 37^{\circ}$ beam-steering range in the E- and H-plane, respectively. The related simulation results are omitted here for compactness.

As we have shown, the AiUC beam-steering performance is power-dependent. At $2.5-\mathrm{dB}$ gain compression regime, the AiUC's E-plane scan range is $\pm 60^{\circ}$ with $2.1 \mathrm{~dB} G_{T}^{c}$ scan loss and $\eta_{D}$ degradation from $47 \%$ to $25 \%$; the $\mathrm{H}$-plane scan range is $\pm 37^{\circ}$ with $1.7 \mathrm{~dB}$ scan loss and $\eta_{D}$ degradation from $47 \%$ to $37 \%$.

\section{Experimental Characterization}

A common approach to experimentally validate array beamsteering performance is to manufacture a relatively large finite array fragment to measure the SEP and active reflection coefficient [22]. Such characterization is usually based on the superposition principle (e.g., [20]) owing to the linearity property of the system. However, as we have discussed previously, this is not applicable to AiAAs in general. The analysis of such arrays requires a full beamforming and feeding circuitry, which can realize the desired excitation of the AiAA [3], [16]. This can be complicated and costly. The waveguide (WG) simulator approach is a good alternative in this case [28]-[30]. This technique has been applied to measure array aperture reflection coefficient [28]. In other studies, the active reflection coefficient is found through the excitation of the single- [29] and multi-element [30] WG simulators, where the WG is terminated with a matched load. The novelty of the present work is the extension of the WG simulator technique to the case of nonlinear and nonreciprocal AiUCs. 


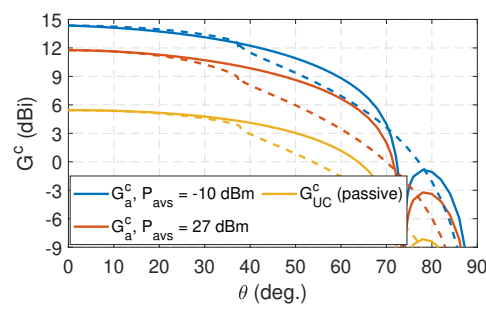

(a)

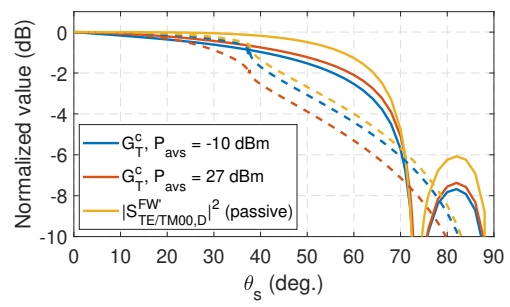

(b)

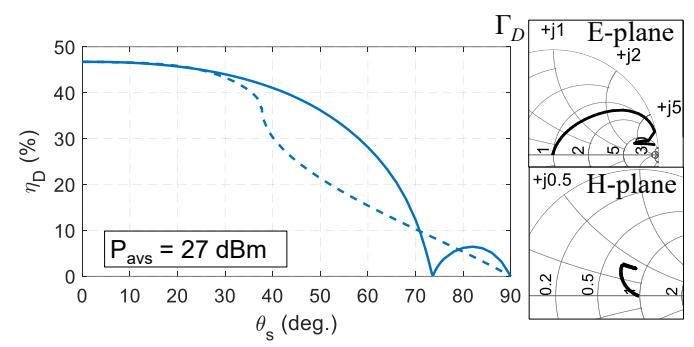

(c)

Fig. 6. (a) Simulated gain active scan element pattern (ASEP) of the full AiUC and scan element pattern (SEP) of the passive radiating part. Computed beam-steering performance of the AiUC versus $\theta_{s}$ : (b) normalized co-polarized AiUC transducer gains (1) and transmission coefficient from the drain port to the dominant Floquet modes; (c) drain efficiency. All results are given at $f_{0}$ in the E- (solid lines) and H-plane (dashed lines) for different $P_{a v s}$. Insets demonstrate $\Gamma_{D}$ variations during beam steering, $Z_{0}=17-j 46 \Omega$.

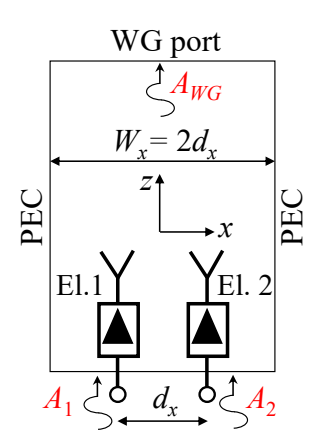

(a)

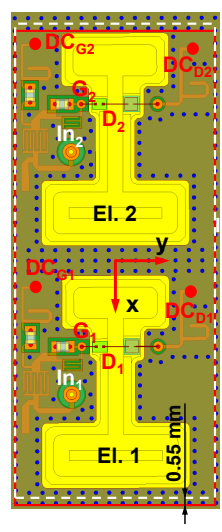

(b)

Fig. 7. (a) The scheme of the two-element H-plane active WG simulator. (b) Top view of the two-element structure inside the WG simulator. The red rectangle indicates WG border, white - geometric border of two AiUCs.

\section{A. Active Waveguide Simulator Technique for AiUCs}

A finite AiAA fragment is placed inside a rectangular WG and excited in such a way that it experiences the same EM conditions as the AiUC at a particular beam-steering regime in the infinite array [28]. We use a two-element H-plane active WG simulator, as schematically shown in Fig. 7(a). The WG transverse sizes are $W_{x}=2 d_{x}$ and $W_{y}=d_{y}$. The following active WG simulator principles are relevant.

1) Each WG mode excitation can be interpreted as the simultaneous excitation of as many as 4 plane waves [28]. If the $\mathrm{TE}_{10}$ mode is excited, the WG simulator provides conditions equivalent to the AiUC H-plane dual-beam operation, with beam steering to $\pm \theta_{s}^{W G}$, where

$$
\sin \left(\theta_{s}^{W G}\right)=\lambda /\left(4 d_{x}\right) .
$$

At $f_{0},(10)$ gives $\theta_{s}^{W G}=23.7^{\circ}$. We then can employ the UC symmetry property [31], resulting in $\Gamma_{D 1,2}=$ $\Gamma_{D}\left(\theta_{s}=\theta_{s}^{W G}, \varphi_{s}=0\right)$, where $\Gamma_{D 1,2}$ denotes the active drain reflection coefficient of the AiUCs inside the WG.

2) The AiUCs should be excited with proper amplitudes $A_{1,2}\left(\left|A_{1,2}\right|=\sqrt{2 P_{a v s 1,2}}\right)$ to generate the desired $\mathrm{WG}$ modal content. This can be formulated mathematically, considering the relations specified in paragraph 1 :

$$
A_{i}=A_{F l}\left[\mathrm{e}^{-j k x_{c i} \sin \left(\theta_{s}^{W G}\right)}+\mathrm{e}^{j k x_{c i} \sin \left(\theta_{s}^{W G}\right)}\right]
$$

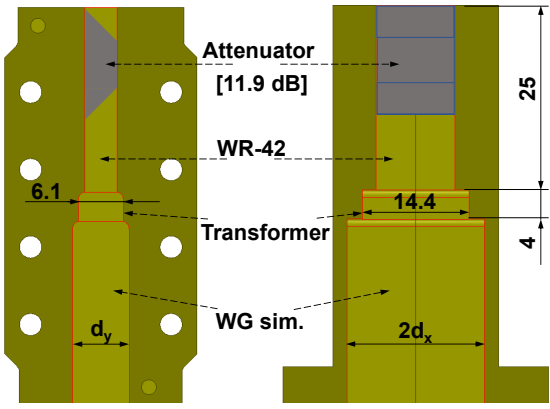

Fig. 8. E- (left) and H-plane (right) cross-sections of the WG structure used to implement the two-element WG simulator. All dimensions are in $\mathrm{mm}$. Fillet radius is $1 \mathrm{~mm}$.

where $A_{F l}=\sqrt{2 P_{a v s}}$ is the magnitude of the incident AiUC voltage wave, providing beam steering to $\pm \theta_{s}^{W G}$; $x_{c i}$ is the $x$-coordinate of the $i$-th AiUC radiation center. Substituting $x_{c 1,2}= \pm d_{x} / 2$ [see Fig. 7(a)], we find $A_{1}=A_{2}=2 A_{F l} \cos \left(k d_{x} \sin \left(\theta_{s}^{W G}\right) / 2\right)$. This result is very important since it ensures that both AiUCs will be under the same compression conditions. Next, the AiUC gains (1) at $\left(\theta_{s}=\theta_{s}^{W G}, \varphi_{s}=0\right)$ can be found through the following relations:

$$
G_{T}^{c}=\frac{\left|A_{W G}\right|^{2}}{2\left|A_{1}\right|^{2}}, G_{P}^{c}=\frac{\left|A_{W G}\right|^{2}}{2\left|A_{1}\right|^{2}\left(1-\left|\Gamma_{i n 1}\right|^{2}\right)} .
$$

Here, we assume that $\Gamma_{i n 1,2}$ are similar. The rigorous derivation of (12) is given in Appendix B.

3) WG sidewalls positions should coincide with AiAA "planes of symmetry" (E-field null-planes) [28]. If the AiUC had been perfectly symmetric in geometry, the radiation and geometric AiUC centers would have coincided. However, the AiUC symmetry with respect to the $y z$-plane is slightly violated due to the different shapes of the upper and lower $y$-oriented slot segments [see Fig.1(b)]. As a result, the AiUC radiation center is shifted relative to its geometric center along $x$-axis by $0.55 \mathrm{~mm}$. To realize the required field symmetry, the WG should cover the structure as shown in Fig. 7(b).

To measure the WG simulator output signal we connected it to the standard WR-42 WG interface through a quarterwave transformer. The longitudinal cross-sections of the full WG structure are given in Fig. 8. The full WG is implemented as two identical blocks connected in the E-plane; the 


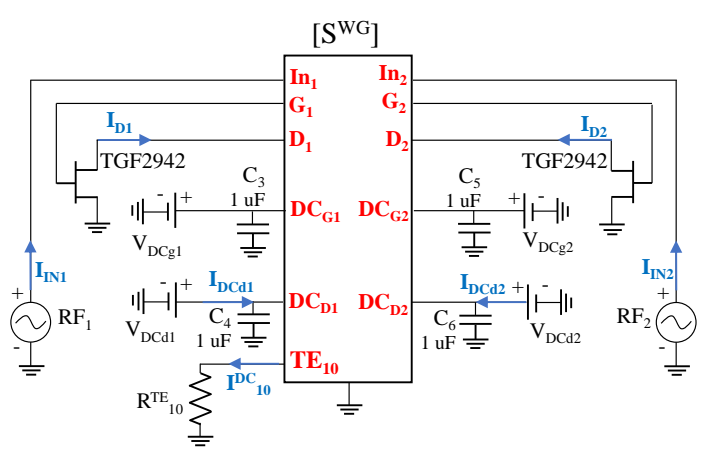

Fig. 9. Circuit diagram of the two-element WG simulator harmonic balance co-simulation setup.

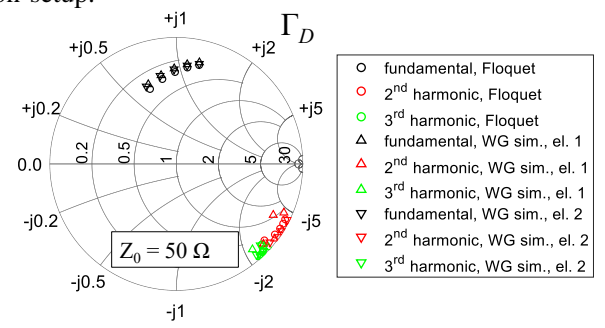

Fig. 10. Comparison of the simulated active drain reflection coefficient $\Gamma_{D}$ at $P_{\text {avs }}=-10 \mathrm{dBm}$ for: the AiUC with periodic boundary conditions for $\left(\theta_{s}, \varphi_{s}\right)=(23.7,0)^{\circ}$, and each AiUC of the two-element WG simulator. Frequency is swept in the $20 \pm 0.25 \mathrm{GHz}$ range with $0.125 \mathrm{GHz}$ step.

WG material is brass. The transformer aims to provide the impedance matching at the fundamental frequency, but it was found to realize a decent matching performance also for the harmonics (reflection coefficient is $-33,-11$ and $-16 \mathrm{~dB}$ at $f_{0}, 2 f_{0}$ and $3 f_{0}$, respectively.). Another discrepancy with the periodic array environment is that the WG simulator, according to (10), provides different $\theta_{s}^{W G}$ at harmonic frequencies. However, since the radiating slot element itself significantly suppresses harmonics radiation, it was found that both abovementioned factors weakly affect the AiUC performance. These assumptions were verified through a co-simulation model. The passive simulator part, comprising the two-element structure covered with the WG, was simulated in HFSS. The ports of the two AiUCs are depicted in Fig. 7(b). A WG port was set at the WR-42 side. The port includes 11 modes propagating at $3 f_{0}$. Next, all higher-order WG modes were terminated with matched loads (Section II-B) resulting in the 11-port $S$-matrix $S^{W G}$. This matrix was then used in the HB co-simulation setup, as depicted in Fig. 9. Controlling the outward power at the $T E_{10}$ port provides the WG simulator output power $P_{W G}=\left|A_{W G}\right|^{2} / 2$. The simulated $\Gamma_{D}$ of the AiUC with $\left(\theta_{s}, \varphi_{s}\right)=(23.7,0)^{\circ}$, and $\Gamma_{D 1,2}$ of both AiUCs inside the WG simulator are presented in Fig. 10. The results are almost identical at the fundamental frequencies, and only a minor discrepancy is observed at $2 f_{0}$. The detailed performance comparison of two models is given below.

\section{B. WG Simulator Prototype and Measurement Setup}

The two-element WG simulator prototype is shown in Fig. 11. The brass base and the WG elements were fabricated by CNC milling; the PCB and all components were mounted using reflow soldering. Gate and drain DC biasing/feeding

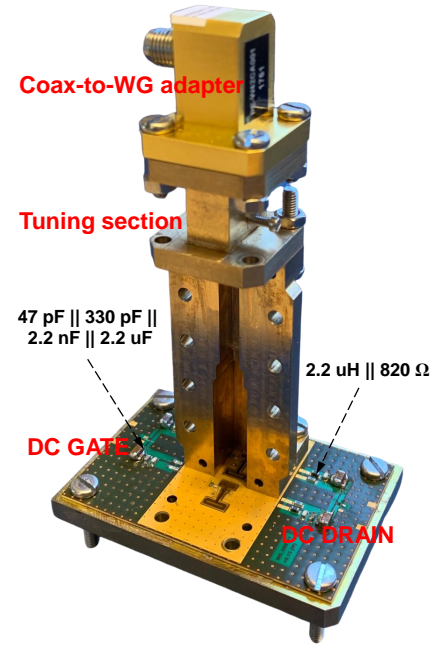

(a)

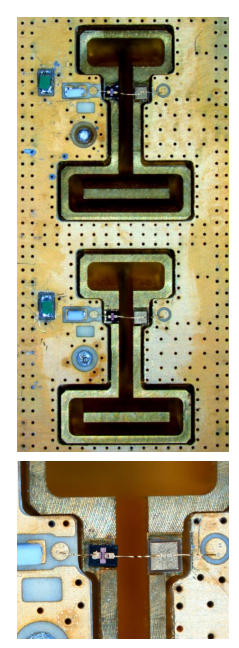

(b)
Fig. 11. (a) Assembled two-element active WG simulator (one half is removed for demonstration). (b) Microscope photographs of the assembled AiUCs.

lines were traces to the peripheral $\mathrm{PCB}$ area with a bank of $47-\mathrm{pF}, 330-\mathrm{pF}, 2.2-\mathrm{nF}$, and 2.2- $\mu \mathrm{F}$ capacitors connected in parallel. The parallel combination of a $2.2-\mu \mathrm{H}$ inductor and a $820-\Omega$ resistor was also introduced in the drain DC circuitry [Fig. 11(a)] for the stabilization and drain current control. The WR-42 interface of the assembly was connected to the measurement equipment through the coaxial-to-WG adapter [Fig. 11(a)]. To compensate for some impedance mismatch of the adapter, which was found to be around $-18 \mathrm{~dB}$, we have employed an additional WR-42 section with two tuning screws placed $\lambda_{0} / 4$ apart [Fig. 11(a)].

First, the small-signal performance, as measured using a vector network analyzer, is demonstrated in Fig. 12. Here we compare the measurement results with the simulated characteristics of the active WG simulator and the AiUC with periodic boundary conditions for $\left(\theta_{s}, \varphi_{s}\right)=(23.7,0)^{\circ}$. We have found that the input matching resonance was initially shifted to the higher frequencies by $0.4 \mathrm{GHz}$. It is believed, and was observed in the simulation model, that the shift was most likely caused by the frequency dispersion and anisotropy of the PCB core laminates. We used an alternative PCB with the length of $T L_{2}$ section increased by $150 \mu \mathrm{m}$. As a result, the frequency shift in $\Gamma_{i n 1,2}$ was partly compensated, although at the cost of a relatively weak peak matching level. Finally, the center frequency of the input impedance matching was measured to be around $20.2-20.25 \mathrm{GHz}$, where the difference between the channels is caused by assembling misalignments. Note that $\Gamma_{i n 1(2)}=S_{1,1(2,2)}^{m}+S_{1,2(2,1)}^{m}$, where $S^{m}$ is the active WG simulator 3-port $S$-matrix as obtained in measurements [Fig. 7(a)]. Additionally, in Fig. 12(a), (b), we have presented transmission coefficients $S_{W G, 1(2)}^{m}$ of both channels separately to support the similarity of their performance. The measured available gain was computed as $G_{P}^{c}=\left|S_{W G, 1}^{m} / \sqrt{1-\left|\Gamma_{i n 1}\right|^{2}}+S_{W G, 2}^{m} / \sqrt{1-\left|\Gamma_{i n 2}\right|^{2}}\right|^{2} / 2$. Predictably, both simulation models demonstrate identical results, with the WG simulator $0.2 \mathrm{~dB} G_{P}^{c}$ drop due to the dissipative loss in the WG structure. The measured $G_{P}^{c}$ 


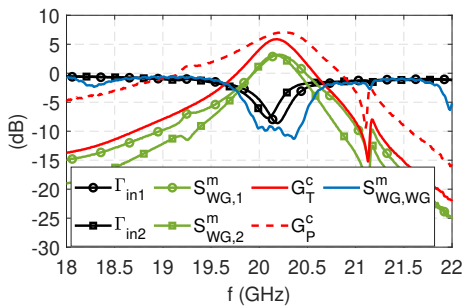

(a)

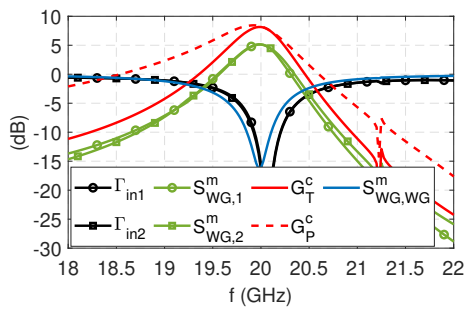

(b)

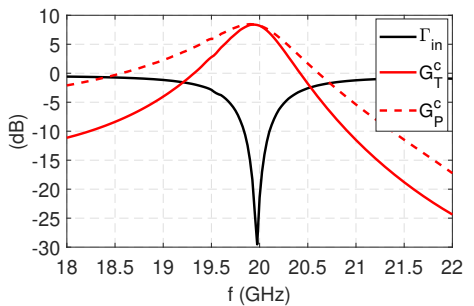

(c)

Fig. 12. Comparison of the small-signal performance. (a) Measured and (b) simulated results of the two-element active WG simulator; (c) simulated results of the AiUC in the infinite array environment for $\left(\theta_{s}, \varphi_{s}\right)=(23.7,0)^{\circ}$.

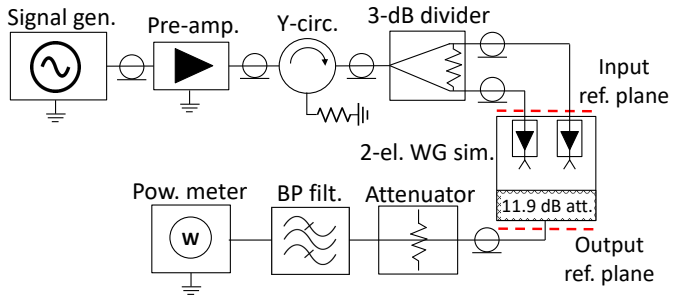

Fig. 13. Two-element active WG simulator large-signal measurement setup.

peak value is $7.2 \mathrm{~dB}$, which is approximately $1 \mathrm{~dB}$ lower as compared to the simulation. Likely, the discrepancy is caused by an additional loss of the WG assembly and input coaxial connectors. Another observation is that the optimum $\Gamma_{D}$ was realized also at higher frequencies, which is indicated by the peak $G_{P}^{c}$ occurring at $20.25 \mathrm{GHz}$ [Fig.12(a)]. The authors believe that this is due to the WG simulator manufacturing and assembling tolerances.

The WG simulator large-signal performance was measured using the setup in Fig. 13. It was found that the coaxial-to-WG adapter does not realize an acceptable impedance matching at harmonic frequencies. To be sure that the adapter will not disturb $\Gamma_{D}$ at harmonics, we introduced an attenuating segment at the output of the WR-42 section (Fig. 8). The attenuator was cut from MAST mf22-0009 foam absorber. Fig. 14 shows a good agreement between the measured and simulated performance.

\section{Results Discussion}

Table I compares the proposed AiUC to the previously reported designs, where at least two amplifying AiAs have been employed. The row "element peak available gain" is defined according to (1) and describes the power amplification performance of the element. When the PA and the radiating element can be characterized separately, e.g. [3], [17], this value equals to the PA gain multiplied by antenna radiation efficiency. When the latter is not reported, only PA gain is given as for [16]. The row "peak active gain" considers both PA and radiating element (or array) gains. For the AiUC, this value equals maximum gain ASEP (Fig. 6). The proposed AiUC co-design approach was found computationally efficient and accurate. It naturally includes array mutual coupling effects and integrated PA behaviour, whereas other methods are either based on a separate radiator and PA design [3], [16] or require a multi-element structure full-wave simulation
[17], [19], [20]. Previously, a somewhat similar approach was demonstrated in [32] where the co-simulation of the active reflectarray unit cell was considered. However, their analysis was limited to the broadside operation regime.

Summarizing the results, we can see that the proposed AiUC is capable of high-power generation with high efficiency at $\mathrm{mm}$-wave frequencies. This performance is realized owing to the high radiation efficiency of the antenna element along with the direct impedance matching. Also, the AiUC demonstrates a relatively wide-angle beam-steering performance, though with a relatively narrow bandwidth, as typical for many AiAs. This is mainly due to poor input HEMT matching and resonant impedance behaviour of the radiating element. The first issue should not be considered as a limiting factor of the AiUC design, as a wideband input matching circuitry can be further implemented together with active components in the MMIC technology. The second factor is more critical, as for improved bandwidth, the antenna element should provide a wideband compensation of a relatively high drain reactance while maintaining almost constant input resistance. At the same time, the EM analysis evidences that inside the targeted bandwidth the radiating slot element behaves as a series resonant circuit, which makes it impossible to realize a wideband optimum loading for the capacitive HEMT drain. Thus, achieving a wide AiUC bandwidth ideally should be addressed through the initial co-design of both radiating and active elements.

\section{CONCLUSION}

The developed amplifying active integrated unit cell (AiUC) offers high mm-wave power generation capabilities with the peak output power of $33 \mathrm{dBm}$ and maximum drain efficiency of $47 \%$ at K-band. This design is capable of providing a wide-angle $\pm 60^{\circ}$ beam-steering range in the E-plane and $\pm 37^{\circ}$ range in the $\mathrm{H}$-plane with the transducer gain scan loss below $2.1 \mathrm{~dB}$ and drain efficiency above $25 \%$ for the $2.5-\mathrm{dB}$ gain compression regime. This result represents a significant improvement over the previously reported designs in terms of the combination of high power efficiency and wide scan range at these frequencies. The main direction of improvement is the relative bandwidth (a few percent in the present case) that is believed can be realized through an initial wideband MMIC PA and radiating element co-design. In this context, the optimum custom impedance at the radiator-PA interface can be found to maximize the bandwidth. 


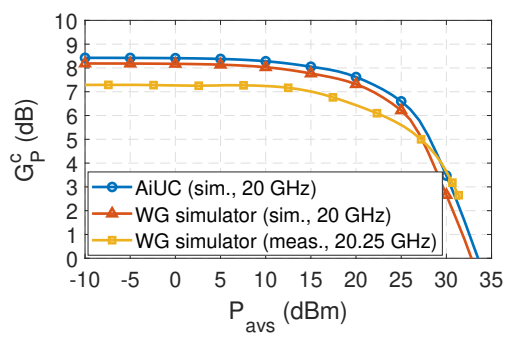

(a)

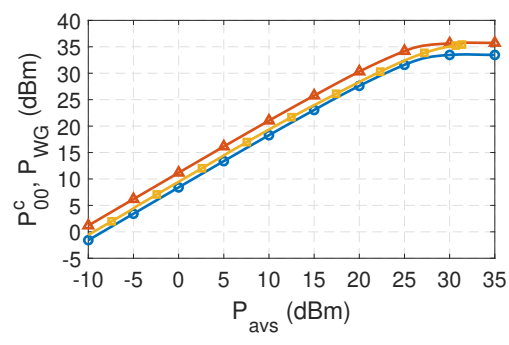

(b)

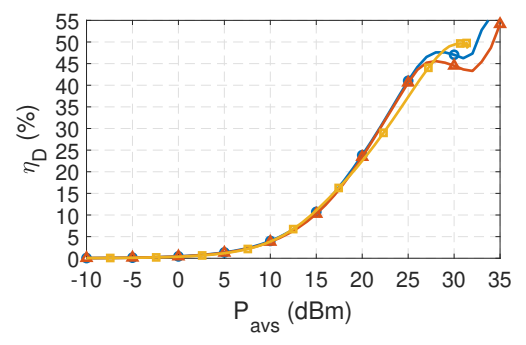

(c)

Fig. 14. Comparison of the large-signal performance for the AiUC in the infinite array environment $\left(\theta_{s}, \varphi_{s}\right)=(23.7,0)^{\circ}$ and two-element active WG simulator: (a) co-polarized available power gain, (b) co-polarized AiUC radiated and WG simulator output powers, (c) drain efficiency.

The proposed AiUC co-design approach has been successfully validated experimentally using the active WG simulator technique; the latter has been extended from its conventional formulation for passive array elements to the case of arrays comprising AiUCs. This represents an important result for designers of active integrated arrays with demanding performance requirements, where the antenna elements mutual coupling and non-linear effects of active electronics play a critical role in the overall system performance.

Future work will extend the proposed methodology to advanced beamforming scenarios where the AiUCs excitation may differ from the considered uniform amplitude distribution (e.g., tapered or MIMO array cases). The corresponding approach is based on the AiUCs mutual coupling analysis [27].

\section{APPENDIX A}

In this study, we use the E-filed Floquet modal functions represented in the following normalized form:

$$
\mathbf{E}_{m n}^{T E}(x, y)=\frac{\mathbf{x}^{0} k_{y n}-\mathbf{y}^{0} k_{x m}}{\sqrt{S Y_{m n}^{T E}} k_{\perp m n}} \mathrm{e}^{-j k_{x m} x-j k_{y n} y}
$$

$$
\begin{gathered}
\mathbf{E}_{m n}^{T M}(x, y)=\frac{\mathbf{x}^{0} k_{x m}+\mathbf{y}^{0} k_{y m}}{\sqrt{S Y_{m n}^{T M}} k_{\perp m n}} \mathrm{e}^{-j k_{x m} x-j k_{y n} y}, \\
Y_{m n}^{T E}=k_{z m n} /(\omega \mu), \quad Y_{m n}^{T M}=\omega \epsilon / k_{z m n}, \\
k_{x m}=k_{x 0}+2 \pi m / d_{x}, \quad k_{y n}=k_{y 0}+2 \pi n / d_{y}, \\
k_{x 0}=k \sin \left(\theta_{s}\right) \cos \left(\varphi_{s}\right), k_{y 0}=k \sin \left(\theta_{s}\right) \sin \left(\varphi_{s}\right),
\end{gathered}
$$

where $k$ is the free-space wavenumber; $\omega$ is the angular frequency; $k_{\perp m n}$ and $k_{z m n}$ are the transverse and longitudinal wavenumbers, respectively [22]; $\epsilon, \mu$ are permittivity and permeability of the free space. The H-field modal functions are defined as $\mathbf{H}_{m n}^{T E / T M}=Y_{m n}^{T E / T M} \mathbf{z}^{0} \times \mathbf{E}_{m n}^{T E / T M}$. Using introduced equivalent surface currents (see Section III-A), the far field can be represented through the wave potentials $\mathbf{A}$, F [26]. After some straightforward but routine mathematical

\begin{tabular}{|c|c|c|c|c|c|c|}
\hline & [3] & [17] & [16] & [19] & [20] & This work \\
\hline Configuration & $2 \times 2$ array & $1 \times 5$ linear array & $2 \times 2$ sub-array & $1 \times 2$ linear array & $1 \times 2$ linear array & AiUC (infinite array) \\
\hline Implementation & $\begin{array}{c}\text { PCB + GaAs } \\
\text { pHEMT; } 50-\Omega \\
\text { match; } 1 \text {-stage PA, } \\
\text { class-E }\end{array}$ & $\begin{array}{c}\text { PCB + AlGaAs } \\
\text { pHFET; } 50-\Omega \\
\text { match; } 1 \text {-stage PA, } \\
\text { class-A }\end{array}$ & $\begin{array}{c}\text { GaAs MMIC; } 50-\Omega \\
\text { match; } 3 \text {-stage PA, } \\
\text { class-AB }\end{array}$ & $\begin{array}{c}\text { PCB + HJ-FET; } \\
\text { direct imp. match; } \\
\text { 1-stage PA, class-A }\end{array}$ & $\begin{array}{c}\text { PCB + HJ-FET; } \\
\text { amplifier-circuit- } \\
\text { antenna concept; } \\
\text { 1-stage PA, class-A }\end{array}$ & $\begin{array}{c}\text { PCB }+ \text { metal base }+ \\
\text { GaN HEMT; direct imp. } \\
\text { matching; 1-stage PA, } \\
\text { class-AB }\end{array}$ \\
\hline$f_{0}, \mathrm{GHz}$ & 2 & 10 & 44 & 10 & 5 & 20 \\
\hline $\begin{array}{l}\text { Bandwidth, \% } \\
\text { (criterion) }\end{array}$ & $\begin{array}{c}22.6\left(\eta_{D} \geq 55 \%\right) \\
13.3\left(3-\mathrm{dB} G_{T}\right)\end{array}$ & $2\left(\left|S_{11}\right| \leq-10 \mathrm{~dB}\right)$ & Not available & Not available & $\left(\left|S_{11}\right| \stackrel{2.5}{\leq}-10 \mathrm{~dB}\right)$ & $\begin{array}{c}4.1,2.6\left(3-\mathrm{dB} G_{P}, G_{T}\right) \\
1.6\left(\left|\Gamma_{i n}\right| \leq-10 \mathrm{~dB}\right)\end{array}$ \\
\hline El. peak av. gain, $\mathrm{dB}$ & 14 & 8.9 & $19.5(\mathrm{PA})$ & 11.5 & 10 & 8.4 \\
\hline Peak active gain, $\mathrm{dBi}$ & $27.5(2 \times 2$ array $)$ & $15.8(1 \times 5$ array $)$ & Not available & $21(1 \times 2$ array $)$ & $18.9(1 \times 2$ array $)$ & 14.2 (single AiUC) \\
\hline Element sizes, $\lambda_{0}^{2}$ & $0.58 \times 0.77$ & $0.8 \times 1.35$ & $0.51 \times 0.51$ & $0.67 \times 0.5$ & $0.33 \times 0.83$ & $0.62 \times 0.51$ \\
\hline $\begin{array}{l}\text { Max. radiated } \\
\text { power/el., } \mathrm{dBm}\end{array}$ & 21.5 & Not available & 9 & Not available & 2 & 33 \\
\hline$P_{1 d B}, \mathrm{dBm}$ & 8.5 & Not available & -11.5 & Not available & -11 & 21 \\
\hline $\begin{array}{l}\text { Peak drain } \\
\text { efficiency, \% }\end{array}$ & $\begin{array}{c}71.35 \\
(6 \mathrm{~dB} \text { compression }) \\
\end{array}$ & Not available & $\begin{array}{c}21.5 \\
(3 \mathrm{~dB} \text { compression }) \\
\end{array}$ & Not available & $\begin{array}{c}20.5 \\
(1 \mathrm{~dB} \text { compression })\end{array}$ & $\begin{array}{c}47 \\
(2.5 \mathrm{~dB} \text { compression }) \\
\end{array}$ \\
\hline $\begin{array}{l}\text { Beam-steering } \\
\text { implementation }\end{array}$ & No (fixed beam) & No (fixed beam) & $\begin{array}{c}\text { Integrated phase } \\
\text { shifters }\end{array}$ & Varactor-tuning & Gate bias variation & External phase shifters \\
\hline $\begin{array}{l}\text { Trans. gain } G_{T} \text { scan } \\
\text { loss and dr. eff. } \eta_{D}\end{array}$ & Not applicable & Not applicable & $\begin{array}{c}1.5 \mathrm{~dB} G_{T} \text { scan } \\
\text { loss }\end{array}$ & $2 \mathrm{~dB} G_{T}$ scan loss & Not available & $\begin{array}{c}2.1 \mathrm{~dB} G_{T} \text { scan loss, } \\
\eta_{D} \geq 25 \%\end{array}$ \\
\hline Beam-steering range & Not applicable & Not applicable & $\pm 26^{\circ}, 2 \mathrm{D}$ & $\begin{array}{c}\text { From }-14 \text { to }+10^{\circ}, \\
1 \mathrm{D}\end{array}$ & $\begin{array}{c}\text { From }+26 \text { to }+30^{\circ}, \\
1 \mathrm{D}\end{array}$ & 2D: $\begin{aligned} & \pm 60^{\circ}, \text { E-plane; } \\
& \pm 37^{\circ}, \text { H-plane }\end{aligned}$ \\
\hline
\end{tabular}
operations, we arrive at the representation (4), where the coefficients $\mathbf{C}_{m n}^{T E / T M}$ have the following forms:

TABLE I

PERFORMANCE COMPARISON OF DIFFERENT TRANSMITTING AMPLIFYING AIAA ELEMENTS 


$$
\begin{aligned}
C_{\theta m n}^{T E}(\theta, \varphi)= & -j \frac{\cos (\theta) k_{z m n}+k}{k_{\perp m n} \sqrt{S Y_{m n}^{T E}}} \times \\
& \left(\sin (\varphi) k_{x m}-\cos (\varphi) k_{y n}\right), \\
C_{\varphi m n}^{T E}(\theta, \varphi)= & -j \frac{k_{z m n}+\cos (\theta) k}{k_{\perp m n} \sqrt{S Y_{m n}^{T E}}} \times \\
& \left(\cos (\varphi) k_{x m}+\sin (\varphi) k_{y n}\right), \\
C_{\theta m n}^{T M}= & -C_{\varphi m n}^{T E}, \quad C_{\varphi m n}^{T M}=C_{\theta m n}^{T E} .
\end{aligned}
$$

\section{APPENDIX B}

To relate the transmission coefficient of the WG simulator to the AiUC gains (1), we can represent the $\mathrm{TE}_{10}$ WG mode as the superposition of two plane waves:

$$
\begin{aligned}
& \mathbf{E}_{W G 10}^{T E}(x)=\mathbf{y}^{0} A_{W G} \frac{\cos \left(k_{W G x} x\right)}{\sqrt{Y_{W G 10}^{T E} S}}= \\
& \mathbf{y}^{0} A_{W G} \frac{\exp \left(-j k_{W G x} x\right)+\exp \left(j k_{W G x} x\right)}{2 \sqrt{Y_{W G 10}^{T E} S}}, \\
& Y_{W G 10}^{T E}=\frac{\sqrt{k^{2}-k_{W G x}^{2}}}{\omega \mu}, \quad k_{W G x}=\pi /\left(2 d_{x}\right) .
\end{aligned}
$$

Each term of (B.1) can be recognized as the Floquet $\mathrm{TE}_{00}$ modal function (A.1) with amplitude $A_{00}^{T E}=\mp A_{W G} / 2$ and $\theta_{s}= \pm \theta_{s}^{W G}$. Next, from (11) we can find the amplitude of the incident AiUC wave exciting each Floquet mode:

$$
A_{F l}=A_{1} /\left(2 \cos \left(k d_{x} \sin \left(\theta_{s}^{W G}\right) / 2\right)\right) .
$$

Finally, substituting (10) into (B.3) and recalling definitions (1) we arrive at (12).

\section{REFERENCES}

[1] K. Chang, R. A. York, P. S. Hall, and T. Itoh, "Active integrated antennas," IEEE Trans. Microw. Theory Techn., vol. 50, no. 3, p. 937-944, Mar. 2002.

[2] S. Gupta, P. K. Nath, A. Agarwal, and B. K. Sarkar, "Integrated active antennas," IETE Tech. Rev., vol. 18, no. 2-3, p. 139-146, 2001.

[3] Y. Qin, S. Gao, and A. Sambell, "Broadband high-efficiency circularly polarized active antenna and array for RF front-end application," IEEE Trans. Microw. Theory Techn., vol. 54, no. 7, p. 2910-2916, Jul. 2006.

[4] A. Khoshniat, T. Yekan, R. Baktur, and K. F. Warnick, "Active integrated antenna supporting linear and circular polarizations," IEEE Trans.Compon., Packag., Manuf. Technol., vol. 7, no. 2, p. 238-245, Feb. 2017.

[5] M. S. Sharawi, S. K. Dhar, O. Hammi, and F. M. Ghannouchi, "Miniaturised active integrated antennas: A co-design approach," IET Microw., Antennas Propag., vol. 10, no. 8, p. 871-879, Jun. 2016.

[6] N. Hasegawa and N. Shinohara, "C-band active-antenna design for effective integration with a GaN amplifier," IEEE Trans. Microw. Theory Techn., vol. 65, no. 12, p. 4976-4983, Dec. 2017.

[7] R. Kumari, A. Basu, and S. K. Koul, "Development of GaN HEMT based high power active integrated antenna," in Proc. 2018 IEEE MTTS Int. Microw. and RF Conf. (IMaRC), Kolkata, Nov. 2018, pp. 1-4.

[8] Y. Lu, Q. Liu, Y. Wang, P. Gardner, W. He, Y. Chen, J. Huang, and T. Liu, "Seamless integration of active antenna with improved power efficiency," IEEE Access, vol. 8, pp. 48 399-48 407, 2020.

[9] Y. Song, Y. Wu, J. Yang, Y. Tian, W. Tong, Y. Chen, C. Wang, X. Tang, J. Benedikt, and K. Kang, "A compact $K_{a}$-band active integrated antenna with a GaAs amplifier in a ceramic package," IEEE Antennas Wirel. Propag. Lett., vol. 16, p. 2416-2419, Apr. 2017.
[10] N. Demirel, Y. Pinto, C. Calvez, D. Titz, C. Luxey, C. Person, D. Gloria, D. Belot, D. Pache, and E. Kerherve, "Codesign of a PA-antenna block in silicon technology for $80-\mathrm{GHz}$ radar application," IEEE Trans. Circuits Syst. II, Exp. Briefs, vol. 60, no. 4, p. 177-181, Apr. 2013.

[11] Y. C. Chen, H. H. Chen, T. G. Ma, and K. Y. Lin, "K-band active antenna integrated with CMOS adaptive-bias power amplifier," in Proc. 2015 IEEE 4th Asia-Pacific Conf. Antennas Propagation, APCAP 2015, Kuta, Indonesia, Jun. 2015, pp. 427-428.

[12] R. Maaskant, O. A. Iupikov, C. A. H. M. van Puijenbroek, W.-C. Liao, M. Matters-Kammerer, and M. V. Ivashina, "Deep integration antenna array: design philosophy and principles," in Proc. 13th European Conference on Antennas and Propagation, (EuCAP 2019), Krakow, Poland, Apr. 2019, pp. 1-5.

[13] H. T. Nguyen, T. Chi, S. Li, and H. Wang, "A linear high-efficiency millimeter-wave CMOS Doherty radiator leveraging multi-feed onantenna active load modulation," IEEE Journal of Solid-State Circuits, vol. 53, no. 12, p. 3587-3598, Dec. 2018.

[14] W.-C. Liao, R. Maaskant, T. Emanuelsson, M. Johansson, A. Höök, J. Wettergren, M. Dieudonne, and M. Ivashina, "A Ka-band active integrated antenna for 5G applications: Initial design flow," in Proc. 2018 2nd URSI Atlantic Radio Science Meeting (AT-RASC), Gran Canaria, Spain, May 2018, pp. 1-4.

[15] W.-C. Liao, R. Maaskant, T. Emanuelsson, V. Vassilev, O. Iupikov, and M. Ivashina, "A directly matched PA-integrated K-band antenna for efficient mm-wave high-power generation," IEEE Antennas Wirel. Propag. Lett., vol. 18, no. 11, p. 2389-2393, Nov. 2019.

[16] H. Wang, M. Aust, K. L. Tan, T. Trinh, C. Liu, R. Esfandiari, and H. C. Yen, "Monolithic Q band active array module and antenna," Applied Microwave, vol. 5, no. 1, pp. 88-102, Winter 1993.

[17] C.-H. Tsai, Y. A. Yang, S.-J. Chung, and K. Chang, "A novel amplifying antenna array using patch-antenna couplers-design and measurement," IEEE Trans. Microw. Theory Techn., vol. 50, no. 8, pp. 1919-1926, Aug. 2002.

[18] S. Kawasaki, "Development of $5.8 \mathrm{GHz}$ high power active integrated antenna arrays," in Proc. 2007 Asia-Pacific Microwave Conference, Bangkok, Thailand, Dec. 2007, pp. 1-4.

[19] S. N. Nallandhigal and K. Wu, "Beam-steered radiation from amplifying active integrated array antenna," in Proc. 2018 48th European Microwave Conference (EuMC), Madrid, Spain, Sep. 2018, pp. 1-4.

[20] — - "Unified and integrated circuit antenna in front end-a proof of concept," IEEE Trans. Microw. Theory Techn., vol. 67, no. 1, pp. 347364, Jan. 2019.

[21] I. Bahl, Fundamentals of RF and Microwave Transistor Amplifiers. Hoboken, NJ: John Wiley \& Sons, 2009.

[22] A. K. Bhattacharyya, Phased Array Antennas: Floquet Analysis, Synthesis, BFNs and active array systems. Hoboken, NJ: John Wiley \& Sons, 2006.

[23] K. Kurokawa, "Power waves and the scattering matrix," IEEE Trans. Microw. Theory Tech., vol. 13, no. 2, pp. 194-202, Mar. 1965.

[24] D. M. Pozar, "The active element pattern," IEEE Trans. Antennas Propag., vol. 42, no. 8, pp. 1176 - 1178, Aug. 1994.

[25] C. Craeye and M. Arts, "On the receiving cross section of an antenna in infinite linear and planar arrays," Radio Science, vol. 39, no. 2, pp. 1-8, Apr. 2004.

[26] R. F. Harrington, Time-Harmonic Electromagnetic Fields. Hoboken, NJ: Wiley-IEEE Press, 2001.

[27] W.-C. Liao, R. Maaskant, T. Emanuelsson, A. Vilenskiy, and M. Ivashina, "Antenna mutual coupling effects in highly integrated transmitter arrays," in Proc. 14th European Conference on Antennas and Propagation, (EuCAP 2020), Copenhagen, Denmark, Mar. 2020, pp. $1-4$.

[28] P. W. Hannan and M. A. Balfour, "Simulation of a phased-array antenna in waveguide," IEEE Trans. Antennas Propag., vol. 13, no. 3, pp. 342 353, May 1965.

[29] M. A. Balfour, "Active impedance of a phased-array antenna element simulated by a single element in waveguide," IEEE Trans. Antennas Propag., vol. 15, no. 2, pp. 313-314, Mar. 1967.

[30] J. J. Gustincic, "The determination of active array impedance with multielement waveguide simulators," IEEE Trans. Antennas Propag., vol. 20, no. 5, pp. 589-595, Sep. 1972.

[31] A. K. Bhattacharyya, "Active element pattern symmetry for asymmetrical element arrays," IEEE Antennas Wirel. Propag. Lett., vol. 6, pp. 275 $-278,2007$.

[32] I. Aryanian, A. Abdipour, and G. Moradi, "Studying the nonlinear performance of an amplifying reflectarray antenna," Int. Journal of Microwave and Wireless Technologies, vol. 9, no. 3, p. 481-491, Apr. 2017. 


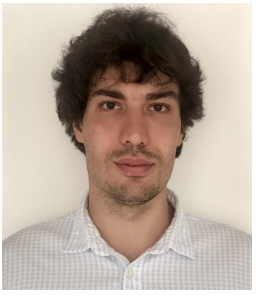

Artem R. Vilenskiy (M'11) received the specialist degree (summa cum laude) in radioengineering and the Ph.D. degree in antennas and microwave devices from Bauman Moscow State Technical University, Moscow, Russia, in 2011 and 2014, respectively.

From 2011 to 2021, he was with Samsung Research Institute Russia, Moscow, Russia, where he had a role of researcher, expert engineer, and project leader in Electromagnetics Group and RF Sensor Part. From 2015 to 2019, he was an Associate Professor at the Radio-Electronic Systems and Devices Department of Bauman Moscow State Technical University. Since 2019 he has been a Researcher with Antenna Systems Group, Chalmers University of Technology, Gothenburg, Sweden. His current research interests include active array antennas, periodic structures, integrated circuits design, controllable high-frequency materials and devices, as well as computational electromagnetics.

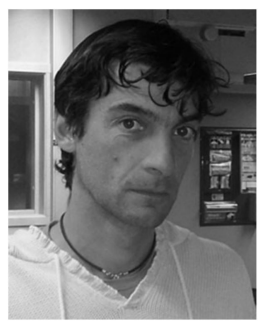

Vessen Vassilev received the M.Sc. degree in radio communications from Sofia Technical University, Sofia, Bulgaria, in 1995, the M.Sc. degree in digital communications and the Ph.D. degree in development of a sideband separating SIS mixer technology for mm-wavelengths from the Chalmers University of Technology, Gothenburg, Sweden, in 1998 and 2003, respectively.

From 1998 to 2008, he was involved in the development of mm-wave receivers for applications in radio astronomy and space sciences. Instruments designed by him are currently in operation at the Atacama Pathfinder Experiment Telescope and Onsala Space Observatory. Since 2008, he has been with the Microwave Electronics Laboratory, Department of Microtechnology and Nanoscience, Chalmers University of Technology. His current research interests include the development of millimeter-wavelength sensors based on monolithic microwave microwave integrated circuit technologies.

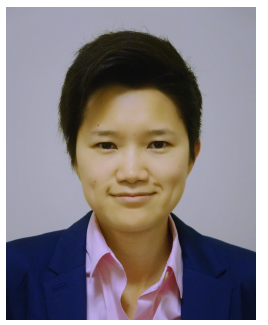

Wan-Chun Liao is currently pursuing a Ph.D. degree in Electrical Engineering at Chalmers University of Technology. Her research interests are high power and high-efficiency active antennas integrated with power amplifiers for large-scale active transmitter arrays and MMIC design.

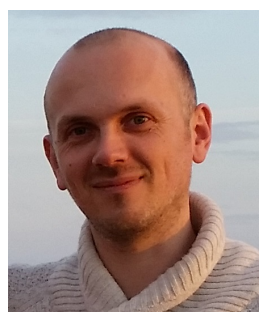

Oleg A. Iupikov (S'12-M'19) received a MSc degree (cum laude) in Electrical Engineering in 2006, from the Sevastopol National Technical University, Ukraine. After graduating, he was working at the Radio Engineering Bureau, Sevastopol. During this period, he was also a visiting researcher at The Netherlands Institute for Radio Astronomy (ASTRON), where he was involved in the development of the focal plane array simulation software for the APERTIF radio telescope. This visit was funded by the SKADS Marie Curie visitor grant and the APERTIF project. Oleg received his $\mathrm{Ph}$. D. degree from the Chalmers University of Technology (Gothenburg, Sweden) in 2017 and currently works there as a researcher.

His research interests are receiving antenna array systems, in particular focal plane arrays for radio astronomy and microwave remote sensing applications, numerical methods for their analysis and optimization, signal processing algorithms for antenna systems, and integration of antennas with active components. He has authored/co-authored over 38 journal and conference papers.

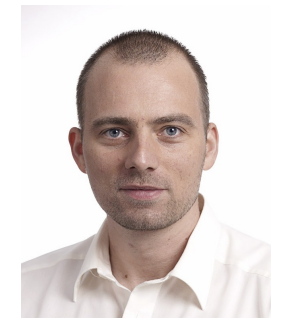

Rob Maaskant (M'11-SM'13) received his M.Sc. and Ph.D. degree (both cum laude) in 2003 and 2010, respectively, both in Electrical Engineering from the Eindhoven University of Technology (TU/e), Eindhoven, The Netherlands. He was employed as an Antenna Researcher at the Netherlands Institute for Radio Astronomy (ASTRON), Dwingeloo, The Netherlands, from 20022010. Since 2010, he has been with the Antenna Group of the Electrical Engineering Department at the Chalmers University of Technology (Sweden), where he was a PostDoc and an Assistant Professor. He is currently an Associate Professor at both Chalmers and the TU/e. The latter position is owing to a 5-year Vidi grant from the Dutch research Council. He is the primary author of the CAESAR software; an advanced integral-equation based solver for the analysis of large antenna array systems. His current research interest concerns the analysis and design of integrated antenna array systems for future wireless applications.

He has served the AP community as an Associate Editor for both the IEEE Transactions on Antennas and Propagation and the IEEE Antennas and Wireless Propagation Letters. He is in the Editorial Board of a unique openaccess journal: Forum for Electromagnetic Research Methods and Application Technologies (FERMAT, http://www.e-fermat.org).

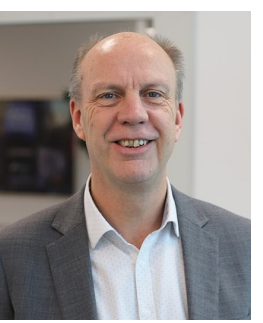

Thomas Emanuelsson received his Master of Science in Electronic Engineering from Chalmers University of Technology in 1984 and is currently holding a position as Expert in Microwave Technology at Ericsson $\mathrm{AB}$ and is an Adjunct Professor at the Microwave Electronics Laboratory, Department of Microtechnology and Nanoscience (MC2), Chalmers University of Technology. Thomas has extensive background in mmWave radio communication, radar phased array systems and MMIC technology. 


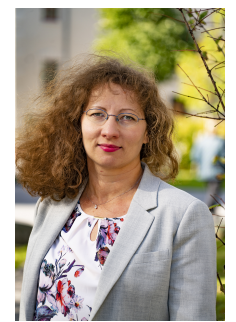

Marianna V. Ivashina (M'11-SM'13) received a $\mathrm{Ph} . \mathrm{D}$. in Electrical Engineering from the Sevastopol National Technical University (SNTU), Ukraine, in 2001. From 2001 to 2010 she was with The Netherlands Institute for Radio Astronomy (ASTRON), where she carried out research on innovative phased array technologies for future radio telescopes, such as the Square Kilometer Array (SKA). In 20022003, she also stayed as a Visiting Scientist with the European Space Agency (ESA), ESTEC, where she studied multiple-beam array feeds for the satellite telecommunication system Large Deployable Antenna (LDA). In January 2011, she joined Chalmers University of Technology (Gothenburg, Sweden), where is currently Full Professor and the Head of the Antenna Systems Group at the Department of Electrical Engineering. Her current research interests include antenna array systems, integration of antennas with active electronic components, synthesis of aperiodic arrays and other unconventional array architectures, reflector antennas, and focal plane array feeds. She has published extensively on the above topics, having authored/co-authored over 130 journal and conference papers. She has received several scientific awards [including the URSI Young Scientists Award for GA URSI, Toronto, Canada (1999), APS/IEEE Travel Grant, Davos, Switzerland (2000), the 'Best team contribution' Paper Award at the ESA Antenna Workshop (2008), the EU FP7 Marie Curie Actions International Qualification Fellowship (2009), Best Paper Award at the IEEE COMCAS Conf., Tel-Aviv, Israel (2019)], and numerous research project funding grants from Swedish and European funding agencies. She is presently an Associate Editor of the IEEE Transactions on Antennas and Propagation and a Board member of the European School of Antennas (ESoA). 